\title{
Comparação de métodos de correção de estande para estimar a produtividade de sorgo granífero ${ }^{1}$
}

\author{
Karla Jorge da Silva ${ }^{2}$, Cicero Beserra de Menezes ${ }^{3}$, \\ Flávio Dessaune Tardin ${ }^{3}$, Vander Fillipe Souza ${ }^{4}$, Crislene Vieira Santos ${ }^{4}$
}

\begin{abstract}
Comparison of stand correction

methods to estimate grain sorghum yield

The magnitute of the experimental error has a direct effect on the success of plant breeding experiments. This study aimed at evaluating the interference of stand correction methods on the yield of 25 grain sorghum hybrids, in seven environments. The methods tested were: without correction, rule of three, covariance of medium and ideal stand, Zuber, Cruz, Vencovsky \& Cruz and stratified correction, based on genotype groups. The Pearson correlation coefficient was used to assess the magnitude of the influence exerted by each method, concerning the data without correction. The stand correction methods based on covariance and Vencovsky \& Cruz reduced the coefficient of variation and showed a high correlation with the adaptability and stability parameters of hybrids, however, these differences were not so significant to justify such adjustment.
\end{abstract}

KEY-WORDS: Sorghum bicolor (L.) Moench; statistic analysis; experimental error.

\section{INTRODUÇÃO}

A magnitude do erro experimental tem efeito direto no sucesso de experimentos, no melhoramento de plantas, sendo que vários fatores interferem nesta magnitude. Os experimentos devem ser conduzidos com o maior rigor possível, para que tenham boa precisão e, assim, as diferenças fenotípicas representem as genotípicas. Há vários fatores que afetam a precisão experimental, como a heterogeneidade do solo, tamanho da parcela, delineamento adotado, número de repetições e condução dos experimentos (Ramalho et al. 2005).

\section{RESUMO}

A magnitude do erro experimental tem efeito direto no sucesso de experimentos, no melhoramento de plantas. Este trabalho objetivou verificar a interferência de métodos de correção de estande na produtividade de 25 híbridos de sorgo granífero, em sete ambientes. Os métodos avaliados foram: sem correção, regra de três, covariância de estande médio e de estande ideal, Zuber, Cruz, Vencovsky \& Cruz e correção estratificada, com base no agrupamento de genótipos. O coeficiente de correlação de Pearson foi utilizado para verificar a magnitude da influência de cada método, em relação aos dados sem correção. Os métodos de correção de estande baseados em covariância e de Vencovsky \& Cruz reduziram o coeficiente de variação e apresentaram alta correlação com parâmetros de adaptabilidade e estabilidade dos híbridos, no entanto, estas diferenças não foram expressivas a ponto de justificarem a correção.

PALAVRAS-CHAVE: Sorghum bicolor (L.) Moench; análise estatística; erro experimental.

A variação de estandes, nas unidades experimentais, é outra dificuldade na análise e interpretação de resultados, em programas de melhoramento de plantas. Por isto, tenta-se obter estandes uniformes pela semeadura em excesso, efetuando-se o desbaste para o estande desejado, quando as plantas estão em estádio inicial de crescimento. Porém, mesmo com esta prática, a parcela pode apresentar falhas, por causa da elevada precipitação pluvial, ou ação de pragas e doenças, o que pode afetar a realização de certas análises estatísticas, em particular quando o caráter não é medido apenas nas plantas competitivas (Vencovsky \& Cruz 1991, Ramalho et al. 2012).

1. Trabalho recebido em ago./2013 e aceito para publicação em jun./2014 (nº registro: PAT 25696).

2. Universidade Federal de Viçosa (UFV), Programa de Pós-graduação em Genética e Melhoramento, Viçosa, MG, Brasil. E-mail:karla.js@hotmail.com.

3. Empresa Brasileira de Pesquisa Agropecuária (Embrapa), Embrapa Milho e Sorgo, Sete Lagoas, MG, Brasil. E-mails: cicero@cnpms.embrapa.br, tardin@cnpms.embrapa.br.

4. Universidade Federal de São João Del Rei (UFSJ), Departamento de Ciências Agrárias, São João Del Rei, MG, Brasil. E-mails: vander_agro@hotmail.com,cris-vieira15@hotmail.com. 
Em ensaios de competição de cultivares, as condições experimentais devem ser as mais uniformes possíveis. São necessários cuidados na implantação, condução dos experimentos e coleta dos dados, a fim de garantir a superioridade de um genótipo, em relação a outro (Carneiro 1998, Cruz \& Carneiro 2003). O uso de princípios básicos no planejamento, condução e análise adequada de experimentos é fundamental para se manter o erro experimental em níveis aceitáveis (Cruz \& Carneiro 2003, Storck et al. 2006). O aumento do erro experimental pode resultar em menor precisão do experimento e reduzir a capacidade de detecção de diferenças entre tratamentos.

$\mathrm{O}$ ajuste do rendimento para o estande ideal, por regra de três simples, constitui um procedimento inadequado, por não existir proporcionalidade linear entre o número de plantas e a produtividade observada, superestimando o rendimento de diferentes genótipos (Ávila \& Sanches 1978, Vencovsky \& Cruz 1991, Schmildt 2000).

Por isso, uma relação mais precisa deve levar em consideração tanto o decréscimo na produção pela presença de falhas, como o acréscimo das plantas vizinhas às falhas, pela ausência de competição (Vencovsky \& Cruz 1991). A fórmula de Zuber (1942) tem sido criticada, porque não leva em consideração a disposição de falhas no campo, e pelo fato de o coeficiente de compensação pela falta de competição ser fixo, independentemente da parcela (Veronesi et al. 1995).

O método de correção dos dados da produtividade de grãos, em experimentos inadequados quanto à população final de plantas, para ser considerado adequado, deve aumentar a discriminação de cultivares, mas não afetar a indicação.

Na utilização de um método de correção, a interferência dessas correções nas estimativas dos parâmetros da análise de adaptabilidade e estabilidade, obtidas pela metodologia de Lin \& Binns (1988), modificada por Carneiro (1998), usada para a indicação de cultivares, é pouco conhecida. Este trabalho objetivou verificar o comportamento da precisão experimental, pelo uso de métodos de correção de estande, em experimentos de avaliação de híbridos de sorgo granífero, em diferentes ambientes.

\section{MATERIAL E MÉTODOS}

Os dados foram originados de ensaios de avaliação de 25 variedades híbridas de sorgo granífero
[Sorghum bicolor (L.) Moench], safra 2011/2012, conduzidos em sete ambientes: Guaíra (SP), Rio Verde e Montividiu (GO), Água Comprida e Sete Lagoas (MG), Teresina (PI) e Vilhena (RO). Destes 25 híbridos, 22 foram desenvolvidos pelo Programa de Melhoramento Genético da Embrapa Milho e Sorgo e os demais foram os híbridos comerciais 1G282, BRS 308 e BRS 330.

As variáveis avaliadas foram o número final de plantas por parcela (estande) e a produtividade de grãos, corrigida para $13 \%$ de umidade, expressa em toneladas $\mathrm{ha}^{-1}$.

As adubações de plantio e de cobertura foram realizadas com base nas análises de solo, e os tratos culturais e fitossanitários de acordo com a necessidade da cultura e de cada região.

Em todos os ensaios, as cultivares foram delineadas em blocos casualizados, com três repetições, com as parcelas experimentais compostas por quatro linhas de $5 \mathrm{~m}$, com espaçamento de $0,5 \mathrm{~m}$ entre as linhas, sendo que apenas as duas centrais foram consideradas como área útil de avaliação e coleta de dados.

As semeaduras foram realizadas a fim de se obterem populações finais de 80 plantas por parcela. As sementes foram distribuídas, uniformemente, em profundidades de 1,0-3,0 $\mathrm{cm}$, sendo realizado o desbaste 30 dias após a emergência. A bordadura do experimento foi plantada com híbridos comerciais. A colheita foi realizada logo após a maturação dos grãos (grãos com, aproximadamente, 15\% de umidade), quatro meses após a semeadura.

Aos dados foram aplicados os testes de Bartlett (para verificar a homogeneidade de variância) e de Lilliefors (para comprovar a distribuição normal), como pressupostos ao uso da análise de variância. Para cada um dos sete ambientes, foram realizadas análises de variância, para as variáveis estande e produção de grãos, e análise de covariância, utilizando-se o estande como covariável. A correção do estande foi feita para os casos em que o efeito estande foi significativo, pelo teste $\mathrm{F}$, a $5 \%$.

$\mathrm{Na}$ análise conjunta, identificou-se o método de ajuste que, para cada ambiente, levou às menores estimativas de coeficiente de variação e maiores estimativas de F. Por isto, foram usados, para cada estatística, princípios da metodologia para adaptabilidade e estabilidade (Lin \& Binns 1988).

A correção da produtividade de grãos foi realizada com o auxílio de oito métodos, conforme Cruz \& Carneiro (2003), em que $Z_{\mathrm{ij}}$ representa a pro- 
dutividade corrigida e $\mathrm{Y}_{\mathrm{ij}}$ o rendimento observado nas parcelas, cujo estande é de $\mathrm{X}_{\mathrm{ij}}$ plantas, e $\mathrm{H}$ representa o estande ideal de 80 plantas, em todos os ambientes, conforme descrito a seguir:

a) Sem correção: os rendimentos foram submetidos à análise, desconsiderando-se a ocorrência de falhas nas parcelas, ou seja, $Z_{\mathrm{ij}}=\mathrm{Y}_{\mathrm{ij}}$;

b) Correção com regra de três: os rendimentos foram corrigidos pela expressão $\mathrm{Z}_{\mathrm{ij}}=\mathrm{Y}_{\mathrm{ij}}$. $\left(\mathrm{H} / \mathrm{X}_{\mathrm{ij}}\right)$;

c) Correção pela fórmula proposta por Zuber (1942): acrescenta 70\% do rendimento médio da parcela por falha, ou seja, $\mathrm{Z}_{\mathrm{ij}}=\mathrm{Y}_{\mathrm{ij}} \cdot\left[\mathrm{H}-0,3 .\left(\mathrm{H}-\mathrm{X}_{\mathrm{ij}}\right)\right] / \mathrm{X}_{\mathrm{ij}}$;

d) Correção pela covariância com o estande médio: o ajuste é, frequentemente, feito por um modelo linear, pressupondo-se que a variação da produtividade dos tratamentos possa ser quantificada pelo mesmo coeficiente: $Z_{\mathrm{ij}}=\mathrm{Y}_{\mathrm{ij}}-\mathrm{b}$. $\left(\mathrm{X}_{\mathrm{ij}}-. . \mathrm{X}\right)$, sendo $b=$ coeficiente de regressão residual de $Y_{i j}$, em função de $X_{\mathrm{ij}}$, estimado conforme o processo descrito por Steel \& Torrie (1980), e ..X = estande médio do ensaio;

e) Correção pela covariância com a população ideal: $\mathrm{Z}_{\mathrm{ij}}=\mathrm{Y}_{\mathrm{ij}}-\mathrm{b} \cdot\left(\mathrm{X}_{\mathrm{ij}}-\mathrm{H}\right)$;

f) Correção utilizando-se a análise de covariância estimada segundo modelo proposto por $\operatorname{Cruz}(1971): Z_{\mathrm{ij}}=Y_{\mathrm{ij}} \cdot\left(\mathrm{H} / \mathrm{X}_{\mathrm{ij}}\right)$ - c $\cdot\left(\mathrm{H}-\mathrm{X}_{\mathrm{ij}}\right)$, sendo $\mathrm{c}=$ coeficiente de regressão residual da variável;

g) Correção por um fator de compensação estimado a partir dos dados experimentais, segundo o modelo proposto por Vencovsky e Cruz (1991): $\mathrm{Z}_{\mathrm{ij}}=$ $\mathrm{Y}_{\mathrm{ij}}$. $\left[\mathrm{H}-\mathrm{a} \cdot\left(\mathrm{H}-\mathrm{X}_{\mathrm{ij}}\right)\right] / \mathrm{X}_{\mathrm{ij}}$, em que $\mathrm{a}=$ coeficiente de proporção e $\mathrm{H}=$ estande ideal;

h) Correção estratificada, proposta por Schmildt et al. (2001): $Z_{\mathrm{ijk}}=Y_{\mathrm{ijk}}-\beta_{\mathrm{k}} \cdot\left(\mathrm{X}_{\mathrm{ijk}}-\mathrm{H}\right)$, em que $\mathrm{Z}_{\mathrm{ijk}}=$ produtividade corrigida para a i-ésima cultivar, na j-ésima repetição, no k-ésimo grupo estabelecido com base na inexistência de diferença significativa entre as médias, conforme teste de Scott \& Knott, realizado ao nível "a" de significância; $\mathrm{Y}_{\mathrm{ij \textrm {k }}}=$ produtividade antes da correção, para a i-ésima cultivar, na j-ésima repetição e no k-ésimo grupo; e $\beta_{\mathrm{k}}=$ coeficiente de proporção do grupo $\mathrm{k}(\mathrm{k}=1,2, \ldots \mathrm{n}$, sendo $\mathrm{n}$ o número de grupos).

A utilização dos métodos de correção de estande visa à redução do erro experimental e ao ajuste da média da parcela. No presente trabalho, a ausência de correção será considerada como um método de correção. Neste caso, a ausência de falhas é ignorada e calcula-se o rendimento da parcela com base na área colhida.

\section{RESULTADOS E DISCUSSÃO}

Os efeitos dos locais e híbridos sobre o estande final foram significativos, mostrando influência dos ambientes em que foram realizados os ensaios, assim como dos diferentes híbridos cultivados (Tabela 1). O teste de agrupamento de média Skott-Knott dividiu os locais em cinco grupos de estande, corroborando a significância do teste F (Tabela 1). Os menores estandes foram observados em Montividiu e Sete Lagoas, com $11,40 \%$ e 12,25\% de redução no número de plantas, respectivamente. $\mathrm{O}$ ambiente com maior estande foi Vilhena, com média de $15,5 \%$ de excesso de plantas (Tabela 1).

Em princípio, irregularidades na distribuição das plantas nas parcelas, responsáveis por modificação na estimativa da produtividade de grãos, são fontes de erro experimental, porque aumentam o valor do quadrado médio do erro, pois, entre as diferentes repetições de um mesmo tratamento, a irregularidade e, por consequência, a produção de grãos não são as mesmas. A irregularidade na distribuição das plantas nas parcelas pode ser efeito da heterogeneidade do

Tabela 1. Médias, coeficientes de variação experimental, quadrados médios de híbridos e do resíduo e teste F, para a variável estande final por parcela, na produção de sorgo granífero, em sete localidades (2011).

\begin{tabular}{|c|c|c|c|c|c|}
\hline \multirow{2}{*}{ Local } & \multirow{2}{*}{ Média $^{1}$} & \multirow{2}{*}{ CV (\%) } & \multicolumn{2}{|c|}{ Quadrado médio } & \multirow{2}{*}{$\mathrm{F}$} \\
\hline & & & Híbrido & Resíduo & \\
\hline Guaíra & $74,6 \mathrm{~b}$ & 14,78 & 162,39 & 121,64 & $2,330 * *$ \\
\hline Rio Verde & $77,5 \mathrm{c}$ & 9,98 & 99,00 & 59,75 & $1,420^{\mathrm{ns}}$ \\
\hline Sete Lagoas & $70,2 \mathrm{a}$ & 11,66 & 107,19 & 66,97 & $1,540^{\mathrm{ns}}$ \\
\hline Teresina & $82,1 \mathrm{~d}$ & 5,41 & 23,18 & 19,69 & $0,330^{\mathrm{ns}}$ \\
\hline Montividiu & 70,9 a & 11,90 & 96,56 & 71,11 & $1,380^{\mathrm{ns}}$ \\
\hline Água Comprida & $73,7 \mathrm{~b}$ & 10,75 & 108,68 & 62,73 & $1,560^{*}$ \\
\hline Vilhena & $92,4 \mathrm{e}$ & 10,05 & 142,33 & 86,22 & $2,040 * *$ \\
\hline
\end{tabular}

${ }^{1}$ Letras iguais, na coluna, não diferem entre si, pelo teste de Skott \& Knot (p >0,05). * e ** significativo a 5\% e $1 \%$, respectivamente; ns não significativo. 
vigor das sementes, diferenças na profundidade de semeadura e aplicação do adubo, condições de umidade para a emergência, ataque de pragas e doenças e plantas daninhas, dentre outras (Vencovsky \& Cruz 1991, Storck et al. 2006). Para contornar este problema, diversos autores têm sugerido o uso de métodos de correção da produtividade das parcelas experimentais como forma de melhorar a precisão experimental (Vencovsky \& Cruz 1991, Veronesi et al. 1995, Schmildt 2000).

A análise de variância individual, referente à característica estande final, nos sete ambientes avaliados, é apresentada na Tabela 1. Os coeficientes de variação $(\mathrm{CV})$ oscilaram de 5,41\%, em Teresina, a 14,78\%, em Guaíra, sendo considerados de baixo a médios, segundo Pimentel-Gomes (2009). Os dados mostraram distribuição normal e homogeneidade de variâncias, podendo todos os ensaios serem avaliados conjuntamente.

Os efeitos dos híbridos sobre esta característica foram significativos, nos ambientes Guaíra, Água Comprida e Vilhena (Tabela 1). A significância ou não, pelo teste F, para a fonte de variação híbridos, não foi influenciada pela amplitude da variação, em relação ao estande ideal. Observou-se, assim, que, tanto Teresina, que não apresentou redução de estande, quanto Sete Lagoas, que apresentou maior redução, foram não significativos, pelo teste $\mathrm{F}$. O mesmo pôde ser observado para o coeficiente de variação: Teresina com menor CV e Montividiu com o segundo maior $\mathrm{CV}$ foram não significativos.

Na tabela 2, é apresentada a análise de variância conjunta, para a produtividade de grãos. A interação híbridos $\mathrm{x}$ locais foi significativa para todos os métodos de ajuste. Isto demonstra que os híbridos responderam de forma diferente à variação ambiental, implicando na importância da análise de adaptabilidade e estabilidade, para a recomendação dos genótipos eventualmente selecionados.

Os ensaios apresentaram valores de resíduos e interação híbridos x locais bem próximos, sendo os mais altos os dos métodos regra de três e Zuber \& Cruz (Tabela 2). A correção pela covariância com estande ideal e a regra de três apresentaram o menor e o maior $\mathrm{CV}$, respectivamente $(13,95 \%$ e $17,35 \%)$. Mesmo assim, a diferença entre estes coeficientes de variação, em comparação ao método sem correção, não foi expressiva a ponto de justificar a correção do estande.

A produção média, em cada ambiente, independentemente das cultivares, após ajuste para cada método de correção (Tabela 3 ), mostrou que a regra de três apresentou os maiores valores em cinco ambientes e levou à superestimação dos resultados. $\mathrm{O}$ sorgo possui compensação do rendimento de grãos, quando submetido à redução aleatória da população inicial, e o componente do rendimento de grãos mais afetado pela redução aleatória da população inicial é o número de grãos por panícula (Montagner et al. 2004). Portanto, similarmente ao milho, na cultura do sorgo, não existe proporcionalidade linear entre o número de plantas e o rendimento observado. Desta forma, o ajuste deste caráter para o estande ideal, por regra de três simples, constitui um procedimento simplório e inadequado, que conduz a resultados superestimados dos rendimentos dos híbridos. Uma relação mais precisa deverá levar em consideração tanto o decréscimo na produção, devido à presença de falhas, como o acréscimo obtido nas plantas vizinhas às falhas, em razão da ausência de produção (Cruz \& Carneiro 2003).

A correção pela covariância com estande médio destacou valores médios de produção similares

Tabela 2. Análise de variância conjunta para produtividade de grãos de sorgo granífero, em sete ambientes, em diferentes métodos de correção de estande (2011).

\begin{tabular}{lrrrrrrrrr}
\hline \multirow{2}{*}{ Causa de variação } & \multirow{2}{*}{ GL } & \multicolumn{10}{c}{ Método de correção $^{1}$} \\
\cline { 3 - 10 } & & SC & RT & \multicolumn{1}{c}{ Z } & COMV & COVI & CRUZ & VC & CE \\
\hline Blocos & 2 & 2,36 & 1,66 & 1,84 & 1,83 & 1,83 & 1,90 & 2,05 & 2,14 \\
Híbridos (H) & 24 & $1,68^{*}$ & $2,04^{*}$ & $1,74^{*}$ & $1,53^{*}$ & $1,53^{*}$ & $1,79 *$ & $1,50^{*}$ & $1,56^{*}$ \\
Locais (L) & 6 & $124,41^{*}$ & $111,10^{*}$ & $113,09^{*}$ & $124,40^{*}$ & $124,24^{*}$ & $133,85^{*}$ & $124,64^{*}$ & $118,88^{*}$ \\
H x L & 144 & $0,87^{*}$ & $1,16^{*}$ & $0,99 *$ & $0,89 *$ & $0,89^{*}$ & $1,01^{*}$ & $0,88^{*}$ & $0,85^{*}$ \\
Resíduo & & 0,40 & 0,60 & 0,48 & 0,36 & 0,36 & 0,45 & 0,37 & 0,38 \\
\hline Médias & & 4,28 & 4,47 & 4,42 & 4,28 & 4,30 & 4,27 & 4,31 & 4,38 \\
CV (\%) & & 14,82 & 17,35 & 15,63 & 14,09 & 13,95 & 15,63 & 14,13 & 14,10 \\
\hline
\end{tabular}

SC: sem correção; RT: regra de três; Z: correção pelo método de Zuber; COVM: correção pela covariância com estande médio; COVI: correção pela covariância com estande ideal; CRUZ: correção pelo método de Cruz (1971); VC: correção pelo método proposto por Vencovsky \& Cruz (1991); CE: correção estratificada. * significativo a $5 \%$, pelo teste $\mathrm{F}$. 
aos sem correção, provocando redução na produção das cultivares que se apresentaram com estande ideal, pois a média dos valores corrigidos deve ser igual à média dos valores observados, portanto, este critério não serve para determinar a adequação de um método de correção.

Os outros métodos obtiveram médias de produção que não se distanciaram muito do sem correção. Observando-se as médias de produção, após cada método de ajuste (Tabela 3), nos ambientes cujo teste $\mathrm{F}$ tenha sido significativo para o estande final por parcela (Tabela 1), observa-se que as correções pela covariância com o estande ideal, pelo método proposto por Vencovsky \& Cruz (1991) e estratificada permitem obter resultados de médias próximas entre si, com certa distinção do método regra de três.

Aplicou-se o índice ambiental (diferença entre a produção média de grãos dos genótipos em cada local e a média geral) como critério para a classificação dos ambientes em favorável (índice ambiental positivo) e desfavorável (índice ambiental negativo). Considerando-se o uso da covariável estande como critério para adequabilidade da correção dos dados da produtividade de grãos, observou-se que os métodos de correção não interferem na classificação dos ambientes, sendo que Rio Verde e Teresina foram favoráveis, com média geral maior que a média geral de todos os ambientes, e os demais foram desfavoráveis, com e sem correção (Tabela 4).

Ao se compararem os postos classificatórios das cultivares nos ambientes gerais (Pig), favoráveis (Pif) e desfavoráveis (Pid), obtidos nos sete métodos de correção da produtividade de grãos, com os dados sem ajuste prévio, o coeficiente de correlação de Pearson variou de 0,775 a 0,995 , o que mostra níveis distintos de concordância na classificação. No melhoramento, buscam-se híbridos com estabilidade geral (Pig), ou seja, aqueles que possuem bom desempenho relativo em ambientes não favoráveis (Pid) e respondem com maiores produtividades, quando da melhoria do ambiente (Pif). Esta metodologia de Lin \& Binns (1988) é muito utilizada por ser de fácil interpretação, em comparação a outras metodologias de estimativas de adaptabilidade e estabilidade. Com relação a Pig, os métodos com maior correlação com os dados sem correção foram a correção pela

Tabela 3. Produção média ( $\mathrm{t}$ ha $\left.{ }^{-1}\right)$ de sorgo granífero, em sete localidades, em razão do método de correção de estande (2011).

\begin{tabular}{lcccccccc}
\hline \multirow{2}{*}{ Local } & \multicolumn{7}{c}{ Método de correção $^{1}$} \\
\cline { 2 - 9 } & SC & RT & Z & COVM & COVI & CRUZ & VC & CE \\
\hline Guaíra & 3,92 & 4,28 & 4,17 & 3,92 & 3,96 & 3,98 & 3,97 & 3,99 \\
Rio Verde & 5,88 & 6,11 & 6,04 & 5,88 & 6,01 & 6,05 & 6,03 & 5,99 \\
Sete Lagoas & 3,26 & 3,80 & 3,64 & 3,26 & 3,28 & 2,96 & 3,29 & 3,40 \\
Teresina & 6,36 & 6,20 & 6,25 & 6,35 & 6,26 & 6,26 & 6,27 & 6,38 \\
Montividiu & 3,24 & 3,67 & 3,55 & 3,24 & 3,47 & 3,50 & 3,48 & 3,47 \\
Água Comprida & 3,82 & 4,19 & 4,08 & 3,82 & 3,92 & 3,92 & 3,93 & 3,94 \\
Vilhena & 3,50 & 3,06 & 3,19 & 3,50 & 3,21 & 3,24 & 3,23 & 3,50 \\
\hline
\end{tabular}

${ }^{1}$ SC: sem correção; RT: regra de três; Z: correção pelo método de Zuber; COVM: correção pela covariância com estande médio; COVI: correção pela covariância com estande ideal; CRUZ: correção pelo método de Cruz (1971); VC: correção pelo método proposto por Vencovsky \& Cruz (1991); CE: correção estratificada.

Tabela 4. Valores dos índices ambientais $\left(\mathrm{t} \mathrm{ha}^{-1}\right)$, em relação à análise conjunta da produtividade de grãos de sorgo granífero $\left(\mathrm{t}\right.$ ha $\left.{ }^{-1}\right)$ (2011).

\begin{tabular}{lrrrrrrrr}
\hline \multirow{2}{*}{ Ambiente } & \multicolumn{7}{c}{ Método de correção $^{1}$} \\
\cline { 2 - 8 } \multicolumn{1}{c}{} & \multicolumn{1}{c}{$\mathrm{SC}$} & $\mathrm{RT}$ & \multicolumn{1}{c}{$\mathrm{Z}$} & \multicolumn{1}{c}{ COVM } & \multicolumn{1}{c}{ COVI } & \multicolumn{1}{c}{ CRUZ } & \multicolumn{1}{c}{ VC } & CE \\
\hline Guaíra & $-0,359$ & $-0,190$ & $-0,108$ & $-0,359$ & $-0,344$ & $-0,297$ & $-0,346$ & $-0,386$ \\
Rio Verde & 1,599 & 1,638 & 1,760 & 1,599 & 1,706 & 1,778 & 1,721 & 1,613 \\
Sete Lagoas & $-0,027$ & $-0,671$ & $-0,645$ & $-1,027$ & $-1,020$ & $-1,313$ & $-1,026$ & $-0,980$ \\
Teresina & 2,073 & 1,728 & 1,964 & 2,073 & 1,961 & 1,988 & 1,954 & 1,995 \\
Montividiu & $-0,043$ & $-0,804$ & $-0,743$ & $-1,043$ & $-0,830$ & $-0,776$ & $-0,835$ & $-0,913$ \\
Água Comprida & $-0,460$ & $-0,286$ & $-0,205$ & $-0,460$ & $-0,383$ & $-0,350$ & $-0,383$ & $-0,444$ \\
Vilhena & $-0,784$ & $-1,415$ & $-1,093$ & $-0,784$ & $-1,090$ & $-1,029$ & $-1,085$ & $-0,885$ \\
\hline
\end{tabular}

${ }^{1}$ SC: sem correção; RT: regra de três; Z: correção pelo método de Zuber; COVM: correção pela covariância com estande médio; COVI: correção pela covariância com estande ideal; CRUZ: correção pelo método de Cruz (1971); VC: correção pelo método proposto por Vencovsky \& Cruz (1991); CE: correção estratificada. 
covariância com estande médio e com estande ideal, método proposto por Vencovsky \& Cruz (1991) e estratificada.

De maneira geral, os métodos de correção por regra de três e Zuber proporcionaram a menor concordância (menores valores do coeficiente de correlação de Pearson) dos parâmetros Pig, Pif e Pid, em relação aos dados originais, quando comparados aos métodos de correção por análise de covariância para a população média, análise de covariância para a população ideal e método de Vencovsky \& Cruz (1991) (Tabela 5).

Ávila \& Sanches (1978) observaram que a correção de rendimento de sorgo pelo método de Zuber (1942), utilizando um coeficiente de compensação estimado no ensaio e aqueles com ajuste pela análise da covariância, foi a mais eficiente. No presente trabalho, a correção por covariância apresentou menor coeficiente de variação e maior correlação com estimativas de estabilidade das cultivares.

Guimarães (2009) comparou esses métodos de correção de estande, na cultura do melão, e concluiu que a correção estratificada foi a mais adequada, pois apresentou baixos valores de coeficiente de variação e altos valores de F. Para o sorgo, a correção estratificada também reduziu o coeficiente de variação, mas não a ponto de se diferenciar de outros métodos de correção.

Tabela 5. Estimativas do coeficiente de correlação classificatório de Pearson, em relação aos parâmetros de estabilidade do método de Lin \& Binns (1988), modificado por Carneiro (1998), entre os dados sem correção da população de plantas e os dados ajustados por diferentes métodos de correção, nos sete ambientes, nos experimentos de genótipos de sorgo granífero (2011).

\begin{tabular}{lccc}
\hline Método $^{1}$ & $\begin{array}{c}\text { Geral } \\
(\text { Pig) }\end{array}$ & $\begin{array}{c}\text { Ambientes } \\
\text { favoráveis (Pif) }\end{array}$ & $\begin{array}{c}\text { Ambientes } \\
\text { desfavoráveis (Pid) }\end{array}$ \\
\hline RT & $0,8726^{* *}$ & $0,7750^{* *}$ & $0,8938^{* *}$ \\
Z & $0,9337 *$ & $0,9134^{* *}$ & $0,9474 * *$ \\
COVM & $0,9768^{* *}$ & $0,9309^{* *}$ & $0,9880^{* *}$ \\
COVI & $0,9768^{* *}$ & $0,9309^{* *}$ & $0,9880^{* *}$ \\
CRUZ & $0,9602^{* *}$ & $0,9234^{* *}$ & $0,9791^{* *}$ \\
VC & $0,9766^{* *}$ & $0,9345^{* *}$ & $0,9877^{* *}$ \\
CE & $0,9440 * *$ & $0,9881^{* *}$ & $0,9954^{* *}$ \\
\hline Média & 0,9487 & 0,9137 & 0,9685 \\
\hline
\end{tabular}

RT: regra de três; Z: correção pelo método de Zuber; COVM: correção pela covariância com estande médio; COVI: correção pela covariância com estande ideal; CRUZ: correção pelo método de Cruz (1971); VC: correção pelo método proposto por Vencovsky \& Cruz (1991); CE: correção estratificada. ** significativo a $1 \%$, pelo teste $\mathrm{t}$.

\section{CONCLUSÕES}

1. Os métodos de correção regra de três, Zuber e Cruz aumentaram os coeficientes de variação e a interação híbridos x locais.

2. Os métodos de correção pela covariância com estande médio e com estande ideal e de Vencovsky \& Cruz reduziram o coeficiente de variação, em relação ao método sem correção, e apresentaram as maiores correlações com parâmetros de adaptabilidade e estabilidade dos híbridos, no entanto, estas diferenças não foram expressivas a ponto de justificarem a correção.

\section{REFERÊNCIAS}

ÁVILA, A. V.; SANCHES, F. M. Comparación de métodos de ajuste para corrección por fallas en sorgos para grano. Agrociencia, Chapingo, v. 31, n. 1, p. 45-64, 1978.

CARNEIRO, P. C. S. Novas metodologias de análise da adaptabilidade e estabilidade de comportamento.1998. $168 \mathrm{f}$. Tese (Doutorado em Genética e Melhoramento) Universidade Federal de Viçosa, Viçosa, 1998.

CRUZ, V. F. Estudo sobre a correção de produção de parcelas em ensaios com milho. 1971. 143 f. Tese (Doutorado em Genética e Melhoramento) - Escola Superior de Agricultura Luiz de Queiroz, Piracicaba, 1971.

CRUZ, C. D.; CARNEIRO, P. C. S. Modelos biométricos aplicados ao melhoramento genético. Viçosa: UFV, 2003.

GUIMARÃES, I. P. Comparação de métodos para correção de estande em experimentos com o meloeiro. 2009. 44 f. Dissertação (Mestrado em Fitotecnia) Universidade Federal Rural do Semiárido, Mossoró, 2009.

LIN, C. S.; BINNS, M. R. A superiority measure of cultivar performance for cultivar x location data. Canadian Journal of Plant Science, Ottawa, v. 68, n. 3, p. 193-198,1988.

MONTAGNER, D.; LOVATO, C.; GARCIA, D. C. Perdas aleatórias na população inicial e sua relação com o rendimento de grãos em sorgo. Revista Brasileira de Agrociência, Pelotas, v. 10, n. 1, p. 81-285, 2004.

PIMENTEL-GOMES, F. Curso de estatística experimental. 15. ed. Piracicaba: Fealq, 2009.

RAMALHO, M. A. P.; FERREIRA, D. F.; OLIVEIRA, A. C. Experimentação em genética e melhoramento de plantas. Lavras: UFLa, 2005.

RAMALHO, M. A. P. et al. Aplicações da genética quantitativa no melhoramento de plantas autógamas. Lavras: UFLa, 2012. 
SCHMILDT, E. R. Correção de rendimento de parcelas, estratificação ambiental e adaptabilidade e estabilidade de cultivares de milho. 2000. 110 f. Tese (Doutorado em Genética e Melhoramento) - Universidade Federal de Viçosa, Viçosa, 2000.

SCHMILDT, E. R. et al. Avaliação de métodos de correção do estande para estimar a produtividade em milho. Pesquisa Agropecuária Brasileira, Brasília, DF, v. 36, n. 8, p. 1011-1018, 2001.

STEEL, R. G. D.; TORRIE, J. H. Principles and procedures of statistics: a biometrical approach. 2. ed. New York: McGraw-Hill, 1980.

STORCK, L. et al. Experimentação vegetal. Santa Maria: UFSM, 2006.
VENCOVSKY, R.; CRUZ, C. D. Comparação de métodos de correção de rendimento de parcelas experimentais com estandes variados: I. Dados simulados. Pesquisa Agropecuária Brasileira, Brasília, DF, v. 26, n. 5, p. 647657, 1991.

VERONESI, J. A. et al. Comparação de métodos de ajuste do rendimento de parcelas com estandes variados. Pesquisa Agropecuária Brasileira, Brasília, DF, v. 30, n. 2, p. 169-174, 1995.

ZUBER, M. S. Relative efficiency of incomplete block designs using corn uniform trial data. Journal of the American Society of Agronomy, Madison, v. 34, n. 1, p. 30-47, 1942. 